\title{
Processing haematological data on a dedicated computer
}

\author{
MG NELSON,* CL FARRINGTON,* JD ROGERS, † AND GW PURDY † \\ From the *Department of Haematology, Royal Victoria Hospital, Belfast, and †Computers Branch, \\ Department of Health and Social Services, Belfast, Northern Ireland, UK
}

SUMMARY A system of processing the laboratory data of a busy haematology department serving a teaching group of hospitals and the general practitioners of the district is described. The system, which has been in routine use for over two years, is based on a dedicated CTL8030 computer sited in the laboratory utilising Lynwood visual display units (VDUs) for both input and access to the data files. Data from the Coulter ' $S$ ' is fed by an interface to the computer and to other data handling modules. Manual test results are entered via VDUs, mainly by laboratory staff who also validate all data. Access is available to all data for answering telephone requests.

Data are retained on the disc file for 48 hours only, and cumulative reporting was not planned into the system as deliberate policy. Quality control of the Coulter ' $S$ ' output is maintained both throughout the day and retrospectively at the end of each working day using both whole blood samples and mathematical treatment of patient data. The output includes printed report documents, patient blood transfusion profiles, a record of blood and product usage, worksheets, quality control information, end-of-day listing in alphabetical order plus statistical information for administrative purposes.

Since its introduction the system has been remarkably free from breakdown as a result of hardware failure. A number of fail safe/fallbacks have been included in the system.

The introduction of automated equipment into hospital laboratories in order to deal with the constantly rising workload inevitably resulted in the production of an enormous amount of test result data. The clerical work involved in handling these data, if carried out by laboratory technical staff, clearly deviates them from their analytical work. The introduction of electronic data processing would improve laboratory performance and allow the development of more sophisticated methods of intralaboratory quality control.

A number of data processing systems have been used in the laboratories at the Royal Victoria Hospital in Belfast. The original system for haematology utilised punch cards and a non-transmitting typewriter. ${ }^{1}$ This was later adapted to produce punch cards, which were used in an off-line batch mode with an IBM 1130 computer which was shared with the Department of Biochemistry at the same hospital. ${ }^{2}$ By 1975 this system presented problems largely as a result of equipment which was nearing the end of its useful life and had become obsolescent.

Received for publication 25 February 1980
A dedicated on-line computer operating in 'real-time' and capable of fulfilling all the data handling requirements of the haematology department was approved, installed, and made operational by November 1977 and has been in routine use since that time. We describe the system at its present state of implementation.

\section{Workload}

The laboratory serves a busy teaching hospital comprising 1807 beds as well as providing a service for 120 local general practitioners. Each year over 100000 specimens are analysed in the routine laboratory with a daily workload of between 350 and 550. In addition, approximately 160000 tests are performed annually in the hospital blood bank, coagulation laboratory, and special haematological biochemistry laboratory, which all form part of the Department of Haematology.

The bulk of the daily workload of the laboratories comes in four or five main batches of specimens collected from the wards by the hospital porters. However, smaller numbers of specimens may arrive at any 
time and include emergency requests which are normally processed immediately on receipt.

\section{Design objectives}

The data processing system for the Department of Haematology was developed with its prime objective towards the service requirements of the laboratory, and it is therefore concerned mainly with capturing, processing, and out-putting laboratory test data. Emphasis is also placed on the use of the computer for quality control. The clerical staff enter the patient identification data (PID) and are responsible for the various printing operations. The input and validation of test result data are, where possible, the responsibility of the laboratory technical staff.

For a total data handling system to gain widespread acceptance from the laboratory staff it must give maximum flexibility. To provide this, the laboratory staff were closely involved in the design phase, and the system was tailored to the needs of the laboratory and was designed so that:

(a) Single specimens, or variable number batches, can be processed and hence allow laboratory staff to operate at their own pace to meet the current workload.

(b) Specimens can be analysed in any order. This ensures that emergency specimens can be analysed without disrupting the entire system.

(c) No restraints are imposed on the order in which the work on each specimen is performed, ie, the input of PID and the various test results are independent of each other.

(d) The laboratory personnel can correct any data, either PID or test results.

(e) Comments on the specimen could, where necessary, replace test results on the final report document.

( $f$ ) As far as possible the computer validates the data, eg, it checks for numeric instead of alphabetic characters. If the input is wrong, it will generate an audible warning and then allow for re-input of the correct data.

Visual display units (VDU) are used for the input of PID and test results and for validation or display of previously entered results. At any time all relevant data up to the most recent are available for display at each VDU in the system. Furthermore, displays of manual test worklists, Coulter ' $S$ ' results, test status, etc, are updated automatically as more recent information (eg, PID) becomes available, without operator intervention being required.

By printing worksheets, hard copies of the data are available for checking or answering telephone queries when the VDUs are otherwise occupied. These worksheets provide an ideal method of out- putting quality control data throughout the day as they are produced as soon as both the PID and Coulter ' $S$ ' results are entered into the system for each group of 19 patient specimens and one control sample. The worksheets also contain an indication of which further tests are required for each sample. Another advantage of producing worksheets is that they provide an extra safety factor to ensure that, on the rare occasions when the computer breaks down, no data already in the system are irretrievably lost. It should be stressed that the worksheets are for convenience only and that they do not impose any constraints on the laboratory staff. Indeed, the system can operate without any worksheets being printed at all.

The system was designed for data handling within the laboratory, and it was felt that most benefit could be derived from the 'real time' nature of the system. However, it was necessary for practical purposes to retain on the computer disc files all the test data for the current day and the previous day. The introduction of cumulative filing would considerably increase the system overheads with timeconsuming disc file searches for information. This would result in a much slower response to commands at all the VDUs in the system unless additional hardware was purchased. Although no comprehensive computer-based cumulative filing is attempted for the routine haematology data, an option in the system provides for limited cumulative filing for selected patients. The primary record will always be the patient's chart, in which all laboratory reports are filed. The actual method of filing using mount sheets (self-adhesive pages with pull-off strips, which leave a sticky strip for fixing the report) results in a cumulative report, the latest report being added to all the previous laboratory data. It was also felt that the clarity of an individual report could be lost when cumulative data are automatically printed.

\section{Hardware}

The computer used is a Computer Technology Limited 8030 with $56 \mathrm{~K}$ words of semiconductor store and a 4.8 million word cartridge disc drive. The disc storage capacity is split with $\mathbf{2 . 4}$ million words on a fixed disc and 2.4 million words on an exchangeable disc. The computer uses a 16 bit word. The CTL 8030 computer is supplied with a suite of programs known as the MODUS IV operating system. This provides a full multiprogramming capability which allows for concurrent multiaccess, through interactive terminals, and batch operation. In practice, this means that at any given time all VDUs in the system can have an application program running, and to the laboratory staff these programs 
appear to function simultaneously. It is aıl important aspect that multiple copies of programs can be active at the same time. Also included in the operating system are disc filing programs, which provide both direct and indexed sequential access to specified named disc files. The computer main frame and disc drive are housed in a separate room, which for operator comfort also requires a small air-conditioning unit. Alongside the main frame is a $\mathbf{2 0 0}$ line per minute Centronics 104 matrix printer, paper tape reader, teletypewriter for giving overall system commands and a VDU. The computer system uses a further five VDUs, two of which are sited in the clerical/reception area and the other three in the routine laboratory itself.

A microprocessor interface, which is necessary to convert the output from the Coulter ' $S$ ' senior into a suitable form (ie, ASCII V24 at 1200 baud) for input to the computer, is made by MSCS Limited, Altona Road, Lisburn, Co. Antrim, Northern Ireland. The interface also provides a second output at 110 baud to a teletypewriter which incorporates a paper tape punch.

Local preference within the laboratory is for the data to be ordered differently from that provided for in the Coulter ' $S$ ' output, so the interface rearranges the order of the results and inserts a decimal point where necessary. A reporter 7, which was originally used to control punching of cards, ${ }^{2}$ is also connected to the Coulter ' $S$ ' but, while it is not essential, it was retained in the new system for its large digital display of each set of results.

\section{Software}

The application programs have been written in the high-level language CORAL, which was specifically chosen because of its suitability for real time systems. CORAL is a structured language producing an efficient executable code, which carries a low overhead relative to that produced by assembly language programs. The application programs were written by staff of the Northern Ireland Department of Health and Social Services, Computer's Branch and by a member of the Haematology Department, Royal Victoria Hospital.

\section{Processing the routine data}

The main sequence of events can be best appreciated by reference to the flow diagram (Fig. 1). When a specimen comes to the laboratory, both the blood sample and the accompanying test request form are given a laboratory accession number. This number is composed of five digits, the first two of which represent the day of the month and the last three

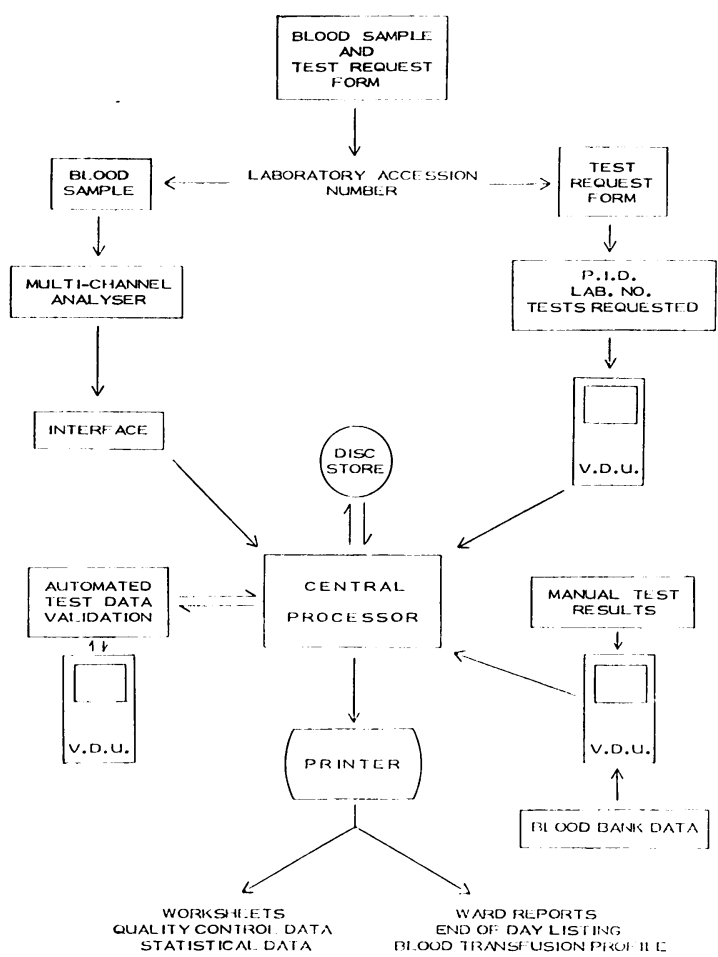

Fig. 1 Flow diagram illustrating computerised processing of laboratory haematological data.

digits start at 001 and are incremented by one for each succeeding specimen. This laboratory accession number is unique in any month and forms the link between the PID and the test results. The computer system is at present designed to cater for up to 800 routine specimens per day but this could be expanded if necessary.

The test request forms are coded for the various tests to be performed on the specimens, although a full blood picture is always assumed unless the request form is coded to the contrary. The PID and test request codes are then input to the computer by the clerical staff through a VDU. If data already exist for the laboratory number, they are displayed and an amend option is given.

The first item of the PID used in the system is normally a hospital number but, to assist the local general practitioners in the identification of their patients, an address may be entered instead. The next fields allow for the input of the patient's surname and initials followed by the age or date of birth. Where the date of birth is entered, the age is automatically calculated to the nearest year but, in the case of young babies, will be correct to the nearest month, week, or day. This is followed by the 
sex of the patient ( $M$ or $F$ ). The next two fields are either a ward code followed by the consultant's initials or, alternatively, for general practitioners the letters GP and the doctor's cypher code number. This cypher number is allocated by the Area Health Authority when the doctor takes up general practice and forms a convenient shorthand for both the doctor and the laboratory.

Lists of the main wards and GPs addresses are retained in the computer on disc files and, by comparing the input data with the codes, a full ward or GPs address can be printed on the final report. These disc files are readily amended with altered or additional codes. Should a test request form arrive in the laboratory with incomplete PID, only the surname of the patient is obligatory, and the rest of the input fields may be left blank.

The last field allows for the input of singlecharacter codes for those manual tests that are to be performed on the sample. Normally, a full blood count is expected for each sample, but, for those requests not requring this analysis, a code letter input at this stage will permit a report to be produced without the full blood count.

The computer also keeps a record of the test status for each sample to show: $(a)$ if the test was requested; and $(b)$ if the test has been completed. This test status information and the PID are available for display on a VDU screen in groups of 20 at any time throughout the day.

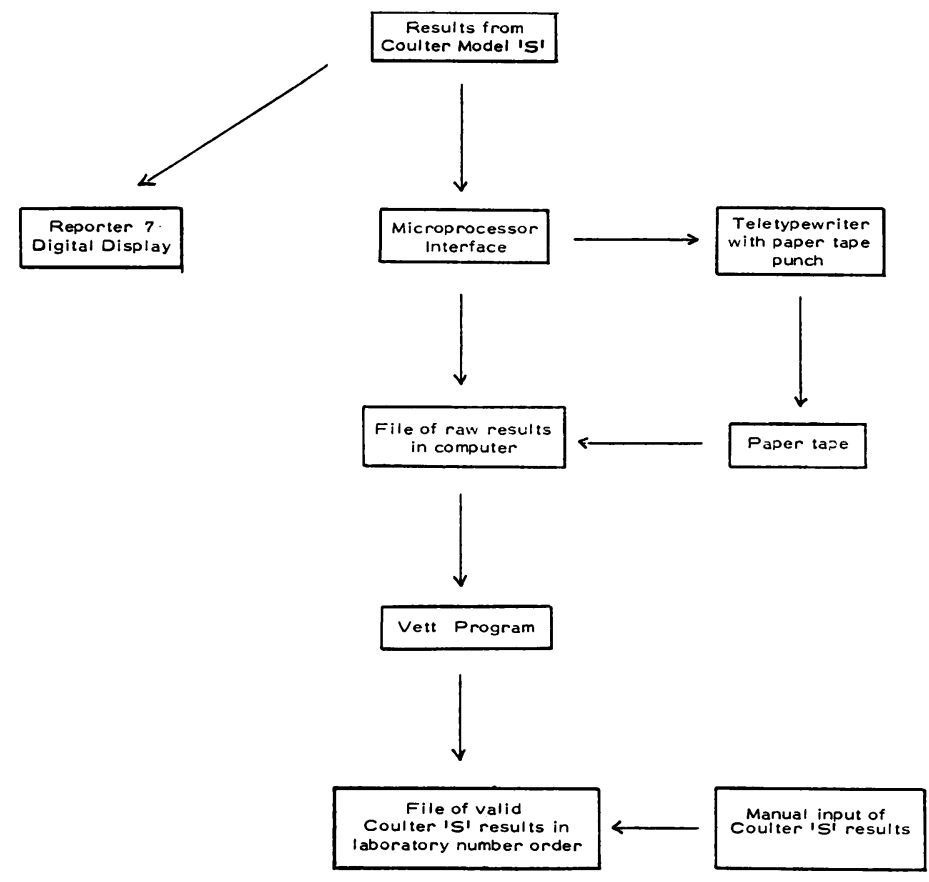

DATA CAPTURE FROM COULTER ' $S$ '

The Coulter ' $S$ ' produces nine pieces of information, namely, a date, a specimen number, and seven haematological parameters on the blood specimen. The date is not used in our system as this is produced by the computer. Each morning an application program is initiated and left running all day, to read the data from the microprocessor interface and to store the results on disc.

Figure 2 shows a flow diagram of the available routes of entry of the data from the Coulter ' $S$ ' to the computer. For normal use, the Coulter ' $S$ ' output is split into two pathways:

(a) to the reporter 7 , which has a large digital display incorporated in the front panel; and

(b) to the microprocessor interface, which relays the results both to the computer and to a teletypewriter to produce a hard printed copy of the data. As the teletypewriter has a paper tape punch facility this means that during periods when the computer is not running, for example, at night, the teletypewriter can also be used to produce a machine-readable form of the data as punched paper tape, which is in turn used for batch input of results, thereby maintaining the integrity of the system.

The system has been designed to place as few restraints on the laboratory staff as possible. Hence the samples can be analysed before the PID is input and vice versa. In addition, there are no restrictions
Fig. 2 Flow diagram showing output of results from Coulter ' $S$ ' and methods of input of data into the computer system. 
on the order in which samples can be analysed by the Coulter ' $S$ ', and each sample can be analysed up to seven times. The only condition is that the specimen number counter on the Coulter ' $S$ ' corresponds to the laboratory accession number on the specimen. As no rigid sampling sequence has to be observed, emergency specimens can be analysed at any time and the results relayed to the wards by telephone.

The computer retains all the results from the Coulter ' $S$ ' in the order in which the specimens were analysed, on a disc file termed the raw data file. However, the results are not necessarily in laboratory number order; some results will be repeated several times and other sets of results will be invalid. Therefore, these raw data cannot be printed on ward reports until they have been checked by the laboratory staff. An application program provides facilities to display these raw data on a VDU screen and for each set of results to be commented on or, if necessary, amended, for example, the addition of a manual white cell count.

Four comment options are available:

1 Accept result: These results are transferred to a separate disc file termed the validated data file. This disc file retains the data in strict laboratory number order and will allow only one set of results per laboratory number. Results which have previously been accepted must be removed from the validated data file before they can be replaced with other results. Provision is also made for examination of the accepted data and for manual entry of results directly on to the validated data file.

2 Delete result: When this comment is made, the data are retained only in the raw data file.

3 Control result: This option provides for the whole blood control results which are produced after every 19 patient results. The data are transferred to a separate file for further statistical analysis.

4 Request further tests: This option enables the laboratory staff, having examined the Coulter ' $S$ ' results, to request further tests, for example, reticulocytes or platelets.

While the samples are being analysed the clerical staff are inputting the PID. As this is a relatively quick procedure (up to three sets of PID per minute can be input by one operator) a laboratory worksheet can be printed as soon as the Coulter ' $S$ ' results are accepted.

QUALITY CONTROL OF COULTER ' $S$ ' RESULTS The quality control program has two components: (a) continuous throughout-the-day monitoring; and (b) comprehensive retrospective end-of-day survey.

For an optimum method of quality control it was felt that both patient sample data and whole blood control specimens sampled regularly should be used.
For this reason, a whole blood control is sampled and a patient sample data algorithm is calculated after every 19 specimens, as recently described. ${ }^{3}$ The calculated results of both the whole blood control and the patient data algorithm are used in the quality control program. Both methods require preset data which are stored on a disc file. The preset data can be examined or altered (eg, mean values for a new batch of whole blood control preparation), or hard copies can be produced on the line printer at any time. To assist the laboratory staff, the system is designed so that when a new batch of whole blood control is prepared, it can be analysed by the Coulter ' $S$ ' with the laboratory number counter set to between 801 and 900 . The results are automatically stored in a special disc file, and subsequently these data can be printed out with a mean and standard deviation for each channel calculated. A further refinement allows for the removal of selected results if necessary.

\section{Throughout-the-day quality control}

There is no substitute for careful monitoring of the patient results by a skilled member of laboratory staff as these are output by the Coulter 'S'. For this reason, a large digital display of each set of results is available beside the Coulter ' $S$ ' on the reporter 7 . Subsequent examination and validation of results through a VDU will show significant trends.

The next stage of the operation is the production of the worksheets which contain the PID and the Coulter ' $S$ ' results for 19 patient samples. At the bottom of this sheet the results of the analysis of the control preparation and the percentage deviation from previously determined means for each channel are printed.

The results of the calculation of the algorithm on patient data for the MCV, $\mathrm{MCH}$, and $\mathrm{MCHC}$, as applied to discrete batches, are also printed. By these methods, direct monitoring of the results and computer-assisted quality control calculations on the data are produced at the time of analysis and provide a good indication of the state of control of the Coulter 'S' (Fig. 3).

\section{End-of-day survey}

At the end of each working day a complete listing of all the control results, percentage deviation from means, and cusums ${ }^{4}$ for all seven channels are printed. In addition to this the $\bar{X}_{B}$ geometric moving mean of Bull and Elashoff ${ }^{5}$ and the Yalgorithm ${ }^{3}$ are calculated for all the patient data, and complete listings for the $\mathrm{MCV}, \mathrm{MCH}$, and MCHC are produced. The computer also keeps a running mean for the patient data which is reset each month and can be compared with the means determined during the previous two years that the system has been in operation. Finally, 


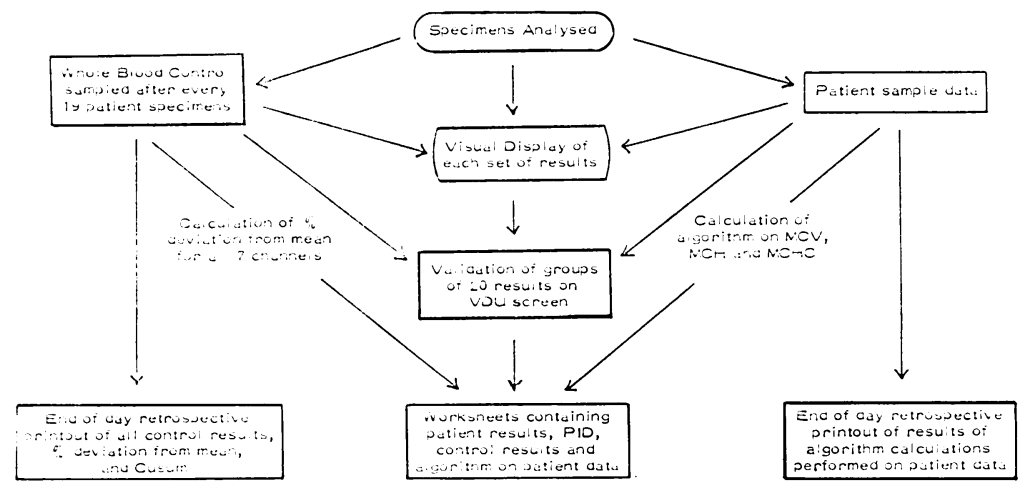

Fig. 3 Flow diagram showing current methods of quality control of the Coulter model ' $S$ ' Senior using the laboratorybased computer system.

while not strictly part of the quality control program, a discriminant function calculation ${ }^{6}$ to discriminate between iron deficiency and a thalassaemia trait is performed on all samples with a MCV less than 80 , and this information is also printed.

MANUAL TEST DATA

The situation for the miscellaneous manual tests (Table) differs from that of the Coulter ' $S$ ' results.

\section{Manual tests catered for in the data processing system}

Differential white cell count

Platelet count

Erythrocyte sedimentation rate

Total eosinophil count

Reticulocyte count

Infectious mononucleosis screening test

Heinz bodies

Lupus erythematosus detection

Leucocyte alkaline phosphatase determination

Blood parasites

The computer automatically expects a Coulter ' $S$ ' result unless an indication to the contrary is entered along with the PID. Hence space on the disc file is allocated for a Coulter ' $S$ ' result for every specimen. However, in the case of the other manual tests, the situation is very different. The specimens that require any such tests are determined in the wards and will vary from day to day. Hence a separate file exists for each test with space in each record for the specimen laboratory number and the test results. In the PID file, each record retains a space for a pointer to every test result, and the pointer will be set if a particular test is requested when the PID is entered. Flexibility is maintained by allowing the test results to be entered before the PID, in which case the program for result entry sets up the pointer in the PID file.

The manual tests are performed by the laboratory staff and the results are entered through a VDU. The program to enter the results displays listings of laboratory numbers, associated PID, and the results of previously completed tests. The laboratory number of the result to be entered is given to the computer, and the Coulter ' $S$ ' data, if available for that laboratory number, are displayed before the computer accepts the result. This sequence of events applies to all manual tests except for the differential white cell count (DWCC).

\section{Differential white cell count}

The program for entry of the differential white cell count displays a worklist of all laboratory numbers requiring the test together with the PID. As results are entered the worklist displays a normal/abnormal indication for tests completed rather than the full differential information. A second part of the program allows input of the differential count on the leucocytes followed by information on the red cell and platelet morphology. Input of the differential white cell count will be considered first. Three basic options are available:

(a) For a normal white cell distribution the result can be reported as 'within normal limits'.

(b) The total numbers of each cell type found can be entered directly.

(c) A small additional keyboard attached to the VDU has the keys coded for 14 different cell types. When a key is depressed, the computer increments the tally for that cell type by one, and displays the new tally. The operator continues to depress the relevant keys for each white cell identified on the slide until the total reaches $\mathbf{1 0 0}$. At this point the computer rings a warning bell in the VDU and will not accept any further results. Erythroblasts are counted but not incorporated in the DWCC, being reported separately.

Provision is made for a total of 15 red cell types, and up to eight may be reported for any one sample. 
The 15 types are displayed on the screen, and the operator indicates which are to be reported by inputting the number(s) allocated to the cell type(s).

Where appropriate, information on platelet number (increased, normal, decreased) and/or size (small, normal, giant) can be printed on the final ward report by the input of a suitable code. A crosscheck on the independently measured platelet count is performed, and a warning is given if the comment on platelet numbers seen on the slide and the total platelet count disagree. Provision is made to indicate that the slide is unsuitable, and a comment to that effect will be reported. A comment is also available for a patient with a white cell count so low that a differential cannot be performed. However, in this case the red cell morphology can be reported. The presence of smear cells and other features occasionally seen on a blood film can be reported by entering a suitable two-character code.

\section{COMMENTS FOR THE REPORT DOCUMENT}

Each day there is a small but significant number of specimens that cannot be processed due to a variety of reasons which include breakages, clots, etc. To cater for these, the system provides for the input of a variety of two-character comment codes. These are split into two categories: those that affect the status of the test, ie, the comment will substitute for a test result, and those comments which will be printed in addition to the test result. An example of the first will be EC for 'ESR specimen clotted', whereas the comment RY for 'received yesterday' will not inhibit the input of a test result.

Up to six comments may be made on any specimen. The comments are all stored on a disc file, and provision is made to allow for changes. For those comments that do not affect the status of the test the list can be added to.

DATA ACCESS DURING THE DAY

It is necessary in any computer system to be able to find test results to answer telephone requests from the wards and other clinical areas. Provision has been made in the system for access to all data for the current day or the previous day by entering one of the three 'keys':

(a) laboratory accession number of the patient specimen;

(b) hospital number of the patient (unique sevendigit number assigned to each patient on admission to the hospital by the admissions office). The computer retains a list of all hospital numbers for patients with data currently on file;

(c) source code; this will normally be the fourcharacter ward code. The computer displays a list of all laboratory numbers and surnames for patients from that source with data on file. For the purpose of this program, the code GP is processed as a single source, and this enables a list of all GP specimens to be displayed on input of this code. By then inputting the laboratory number for the patient concerned all the test data currently available are displayed.

REPORTS AND END-OF-DAY LISTING

The computer system places no constraints on the laboratory staff regarding the order in which test results can be input. However, it will not normally print final report documents until all result data for tests requested have been input. In practice, this means that because an indication of the tests requested is input along with the PID, reports cannot be printed until each test requested has a result input.

The program to print the ward reports sorts through all the laboratory data and prints only those reports that are complete and have not been previously reported. Reports with tests incomplete are delayed, but a list of both reported and unreported laboratory numbers is generated after each reporting run. Provision is made to override the normal checks and to print either previously reported or incomplete reports. It is also possible to print reports in destination order.

In addition to the test results and the PID, the report document contains sufficient information for it to be 'homed' to the correct destination. For the Royal Victoria Hospital complex this will be a ward description and an indication as to whether the report is for the main, maternity, or children's hospital. Reports for the general practitioners will contain the doctor's name and surgery address. (Many GPs have more than one surgery, and the report must be addressed to the correct one.) As it proved impossible to contain the results from a full blood picture and the data from an abnormal white cell count on the same report, a second report is printed for an abnormal differential white cell count. (The DWCC is classed as abnormal if cell types not usually found on a normal smear are seen or if more than five red cell morphology types are reported.)

While the reporting program will print only reports that are complete, in practice it has been found that the routine of the laboratory is best served by three printing sessions in the day. Consequently, reports are usually printed ready for dispatch to the wards a maximum of 3-4 hours after receipt of the specimen.

Each evening an end-of-day listing is produced containing all test results, and the associated PID are sorted into alphabetical order of the patients' surnames. This listing is then retained in the laboratory 
as a record of the day's work and to answer subsequent requests for results. A duplicate listing containing only the data from specimens from general practitioners is produced.

To obtain statistical information on the laboratory workload for administrative purposes, the program used to produce the alphabetical listing also updates monthly totals for each test performed. The test workload for the day is then printed as well as the running totals for the month. Once the day's listing is produced, the computer files can be cleared ready for the next day's work. However, it is most unusual to find that all tests have been completed for all the samples on any one day. To allow for this, the system has been developed so that up to 200 samples may be carried over for completion the next day.

BACK-UP AND OUT-OF-HOURS WORKING

The normal practice in the department is to run the computer system only during working hours. To deal with out-of-hours test results, batch mode processing is used with punched paper tape of the Coulter ' $S$ ' results and manual entry of all other data from the request form which can double as a report document. This is also the system used when the computer is out of operation for a limited period for a minor breakdown or maintenance. The problems arising from equipment failure will, to a large extent, depend on the module or modules that break down.

1 The teletypewriter with paper punch and reporter 7

These modules both provide very useful facilities and a fall-back capability but, in the event of breakdown, will not prevent the rest of the system from functioning normally.

2 The computer and disc drive

It is obvious that the efficiency of this system is largely dependent upon the reliability of the computer. In the two years of routine operation there have been only two occasions when the day's workload has not been completed through the system. Generally any faults that have appeared have either been rapidly fixed by a service engineer or have permitted operation to proceed with a reduced capability. Where necessary, it is possible to resort to an entirely manual system.

3 The interface

Failure of this module would necessitate the manual entry of the Coulter ' $S$ ' data.

4 The printer

When the breakdown is of short duration, all printing operations are delayed until the printer is operational again. In the event of a prolonged failure, all printing could be performed by taking the exchangeable disc from the disc drive to the Biochemistry Department for printing by their CTL Mod 1 computer system.
Processing the hospital blood bank data

The work of the hospital blood bank can be conveniently split into two main sections. The first deals with the $\mathrm{ABO}$ and Rhesus (anti-D) grouping of patient blood samples and an antibody screen for the presence of irregular erythrocytic, platelet, or leucocytic antibodies in the patient's serum. The second category is concerned with cross-matching and issuing blood for transfusion. Apart from issuing single donor units of blood, the hospital blood bank is also responsible for issuing blood products and commercial concentrates.

BLOOD GROUP AND ANTIBODY DATA

The PID and the results of the laboratory tests are entered manually on a preprinted worksheet which is kept in the laboratory work area. This is used to record the results of blood grouping or antibody screening tests. The data are taken from these handwritten worksheets and entered into the computer via a VDU. The program for entry of these data requests the PID followed by the test results. Provision is also made to enter the results of a direct Coombs' test and an antibody screen. If either of these last two is positive, the computer allows for the entry of further data on the nature and type of antibody found. A comprehensive list of antibody types is retained on a disc file and is accessible for amendment if necessary. The need to obtain information about the actual administration, rather than issue, of blood products led to the introduction of a triplelabel system using NCR paper. This triple label is completed by the member of the laboratory staff responsible for cross-matching the blood or issuing the blood products. One of these forms is retained in the laboratory and used as a source of information for input to the computer and to signify that the blood/blood product has been issued. The other two forms are attached to the blood/blood product. When the blood/blood product is returned unused to the blood bank, both forms are still attached. However, if the blood/blood product is used, only one of the forms is returned to the blood bank, the other being retained in the patient's chart.

The computer system starts with the entry of the data on the blood/blood products issued. Details of the patient and information on the type of blood/ blood product, unit or batch number, cross-match results, technician's initials and, where applicable, the units of clotting activity or volume are entered. It is also possible to indicate where non-homologous blood was used. A complete listing of the blood/ blood product issued is printed daily and used as a permanent record in the blood bank. In order to maintain a blood bank stock control system, and to 
enable a patient's blood transfusion profile to be prepared, the blood/blood product information is stored by the computer on a disc file for one month.

The next stage of the system displays on a VDU screen a listing in ward order of all the blood issued but unaccounted for. Using the returned forms, the operator is able to go down the lists and comment 'used' or 'not used' for the blood/blood product. The unused blood is deleted from the file. The used blood is transferred and stored under the patient's name on a separate file.

Normally the blood grouping data are entered in batches of 20-40 sets of results. After data entry, the blood group report documents are printed and the data are cleared from the computer disc file. In certain circumstances, where a direct Coombs' or antibody screen is positive, the results of the investigation of the antibody present are not immediately available. In this case, the incomplete data are retained, and each time the program is used a reminder is displayed on the VDU screen.

For patients with a positive direct Coombs' test or antibody screen, a duplicate report document is printed. These duplicate reports are filed in the laboratory for future reference.

The ward reports are validated against the handwritten worksheet and colour-coded according to the patient's blood group. Where antibodies are present, a red warning label is also attached to the report document.

BLOOD AND BLOOD PRODUCT DATA

In addition to the issue of whole blood and blood fractions supplied by the Regional Blood Transfusion Service, the hospital blood bank issues about 15 commercial products, including plasma protein fractions and concentrates of coagulation factors. As the list of commercial products is subject to change it is retained on a disc file, which contains a blood/ blood product description and a code. For the coagulation factors and volume replacement products the ranges of the clotting activity or volume are also stored. This ensures that changes to the products do not result in any reprogramming.

Approximately once a month a patient transfusion profile for all patients who have been receiving blood/ blood products during the previous month is printed for inclusion in the patient's chart. This patient transfusion profile should be particularly useful in the maintenance of treatment statistics for patients with hereditary bleeding disorders, particularly the haemophiliac patients who attend the Regional Centre. This provides readily accessible information on the amount of cryoprecipitate or commercial concentrates used.

During the month these data will be accessible at a
VDU, and at any time ward lists of all blood unaccounted for can be printed for each ward in the hospital. The system has been designed to enable the maintenance of a list of patients who have been transfused with each different batch of commercial concentrate. The data can either be displayed on a VDU screen or printed.

\section{Processing data for the coagulation and haematology/biochemistry laboratories}

Both these laboratories use the computer facilities for processing the data from the most common tests performed, namely, the Thrombotest and prothrombin time for the coagulation laboratory and the serum iron, total iron binding capacity (TIBC), and ferritin concentration for the haematology/ biochemistry laboratory. The systems for the two laboratories follow similar lines.

On receipt of the specimen both the sample and test request form are given a laboratory number. The test request form is then given to the clerical staff for input of the PID. While the PID is the same as for the routine laboratory, indexed files are used with the laboratory number as the 'key'. Subsequent data access is always achieved by using the laboratory number. When the PID is entered a printed worksheet in laboratory number order is produced. The PID can be verified against the worksheet for amendment if necessary.

As none of these tests has an automatic digital output, being either manual or having an output in the form of a peak on a continuous chart, the results are usually manually written on to the worksheet for subsequent entry via a VDU into the computer. When the results are entered, reports are printed. At the end of the day an alphabetical listing is produced for retention in the laboratory.

The coagulation laboratory normally produces the result of the Thrombotest and prothrombin time in seconds but reports them as a percentage of normal. The computer uses a disc file to store a table of times and percentage and automatically performs the conversion from seconds to percentage. However, to enhance the flexibility, the percentage result can be directly entered and no conversion is performed. Both the time in seconds and the percentage are reported for the prothrombin time but only the percentage for the Thrombotest.

The table used for conversion of time to percentage is a feature of each batch of reagent used for the tests, and a separate program allows the laboratory staff to change the table when necessary. The haematology/biochemistry laboratory usually produces the results of the serum iron and TIBC analyses several times each week but the ferritin estimations 
only once. As each sample requiring a ferritin result will always have a serum iron and TIBC result, these samples must have the data retained until the ferritin result is available. Normally, the serum iron and TIBC results are printed on an interim report as soon as they are available, and a second report is printed when the ferritin analysis is performed.

As the ferritin concentration is measured using a radioimmunoassay the computer is used to assist in the calculation of the results. The method currently in use is a linear interpolation between points, but a polynomial regression method is being developed. The standard concentrations and counts are entered, and the computer then calculates the slope of the line between each pair of points. As the unknown results are entered the computer decides between which pair of standards the result lies and uses the relevant slope to convert the count into a concentration. The duplicate result is treated in the same way and, if the difference between the two results is less than $5 \%$, a mean value is calculated and printed, otherwise both results are printed out.

\section{Cost and benefits}

The hardware that was purchased in 1977 cost approximately $£ 40000$ with an annual expenditure of $£ 4800$ for equipment maintenance and a further $£ 2000$ for paper and other consumable items. Software development was all performed by National Health Service and Department of Health and Social Services staff and took several man-years.

The routine operation of the system requires a clerical staff of two in addition to the normal laboratory technical staff. However, it is possible that without a computer system further laboratory staff would have been required.

There has been widespread acceptance of the system by all involved. This is due in part to the close liaison with the laboratory technical staff during the design phase. The computer system now forms an integral part of the whole laboratory operation. The greatest benefit of the system is in an improved workflow, and hence considerable savings in time were noticed when it went into operation. The main areas of benefit are probably in:

1 the reduction in clerical effort by the laboratory staff;

2 accessibility of test data both current through the computer and previous results in the form of concise alphabetical listings;

3 improvement in the time taken to perform differential white cell counts;

4 quality control of the Coulter ' $S$ ' improved by the production of current data without tedious calculation by the operator;

5 statistical information on workload, blood/blood product usage, etc, readily available.

For the clinician the improvements are probably a better format of printed report document, rapid reporting, and a faster response to telephone requests. The patient transfusion profile has proved to be a valuable record, particularly for multitransfused patients and patients with coagulation defects.

\section{References}

1 Nelson MG. Punch card data processing in haematology. $J$ Clin Pathol 1969;22:233-5.

2 Nelson MG, Elder E. Data processing in haematology. $J$ Clin Pathol 1972;25:594-8.

${ }^{3}$ Lappin TRJ, Farrington CL, Nelson MG, Merrett JD. Intralaboratory quality control in haematology. Comparison of 2 systems. Am J Clin Path 1979;72:426-31.

4 Cavill I, Ricketts C. Automated quality control for the haematology laboratory. Cusum method. J Clin Pathol 1974;27:757-9.

${ }^{5}$ Bull BS, Elashoff RM. Preprint. Proceedings of the San Diego Biomedical Symposium 1974;13:1-5.

- England JM, Fraser PM. Differentiation of iron deficiency from thalassaemia trait by routine blood count. Lancet 1973 ; i 449-52.

Requests for reprints to: Dr CL Farrington, Department of Haematology, Royal Victoria Hospital, Belfast BT12 6BA. 